\title{
Preface to the special issue on inverse problems
}

\author{
Jin Cheng • Yiu Chung Hon • Masahiro Yamamoto
}

Published online: 12 June 2010

(C) Springer Science+Business Media, LLC 2010

The investigation of inverse problems is important due to its significance in practice to the real application fields such as medical diagnosis, materials sciences, geophysics, and industrial engineering. The recent rapid development in numerical methods for solving inverse problems has been attracting much attention from mathematicians, engineers, and scientific researchers. Inverse problems are in nature concerned with the detection of hidden "reasons" from incomplete observations of "outcomes". In practice, most of these detections, testings, and diagnoses possess the character of inverse problems: intrinsic instability. In view of the significance in practical applications, exploitations of feasible and robust numerical methods that can handle well the instability of inverse problem are indispensable. Some of these numerical methods have been exploited due to the pioneering "regularization" work by Tikhonov. Witnessing the recent rapid development in computational technologies and measurement equipments, we are now at the edge towards the next stage when more variety of numerical methods can be developed for real and applied inverse problems. Moreover, with the substantial accumulation of theoretical results for inverse problems, novel numerical methods can be invented.

Communicated by guest editors: Jin Cheng, Yiu Chung Hon and Masahiro Yamamoto.

J. Cheng

School of Mathematical Sciences, Fudan University, Shanghai, China

e-mail: jcheng@fudan.edu.cn

Y. C. Hon $(\bowtie)$

Department of Mathematics, City University of Hong Kong,

Hong Kong SAR, China

e-mail: maychon@cityu.edu.hk

M. Yamamoto

Department of Mathematical Sciences, The University of Tokyo, Tokyo, Japan 
The special issue contains seven papers showing such novel numerical methods as follows:

"Adaptive wavelet methods and sparsity reconstruction for inverse heat conduction problems" by T. Bonesky, S. Dahlke, P. Maass and T. Raasch discusses an inverse heat conduction problem which is very important e.g., for monitoring plants in engineering, and combines an adaptive wavelet algorithm with the regularization technique to show confirming numerical results.

"Numerical pseudo-differential operator and Fourier regularization" by C.-L. Fu and Z. Qian discusses the numerical pseudo-differential operators to which many inverse problems are reduced, and proposes the Fourier regularization for overcoming the instability. Theoretical analysis is performed and examples are tested.

"Discretized Tikhonov regularization by reproducing kernel Hilbert space for backward heat conduction problem" by Y.-C. Hon and T. Takeuchi treats a backward heat conduction problem which is another typical inverse problem for the heat equation. The authors propose flexible discretization on the basis of the reproducing kernel for the standard Tikhonov regularization, and the flexibility in controlling the discretization accuracy is a key for overcoming the instability and convincing numerical examples are given.

"Numerical method for the inverse heat transfer problem in composite materials with Stefan-Boltzmann conditions" by X. Hu, X. Xu and W. Chen considers a specific kind of heat transfer problem with nonlinear StefanBoltzmann interface conditions in multi-layer materials in manufacturing industries. Although such interface condition appears frequently for a heat process modelling, there are very few mathematical researches. The paper establishes theoretical results for the forward problem and an inverse problems of determining corrosion part, and numerical simulations for both the problem.

"Huygens' principle and iterative methods in inverse obstacle scattering" by O. Ivanyshyn, R. Kress and P. Serranho considers an inverse problem of determining the shape of an obstacle from the knowledge of the far field pattern for scattering of time-harmonic plane waves. In the paper, the authors apply methods by integral equations and propose three iteration methods. They then clarify the relations among these three solution methods and relate them with the traditional regularized Newton type iterations.

"Convergence analysis for the Cauchy problem of Laplace's equation by a regularized method of fundamental solutions" by T. Wei and D. Y. Zhou discusses the Cauchy problem of Laplace's equation which is also a very classical inverse problem and appears in various cases. The authors combine the method of fundamental solutions with the discrete Tikhonov regularization to achieve robust numerical results against noisy Cauchy data. Under the suitable choices of a regularization parameter and an a priori assumption on the solution, a convergence result for the regularized solution is proved and numerical experiments are presented. 
"Stable numerical differentiation for the second order derivatives" by H. $\mathrm{Xu}$ and J. Liu considers a numerical differentiation problem, which aims to compute stably the second order derivative of a function from given noisy data. For this ill-posed problem, they use the Lavrent's regularization. The a priori and a posterior choice strategies for the regularization parameter are considered and convergence and error estimates for the regularizing solution are proved with convincing numerical examples. 